\title{
Deformidad de Gibbus no tuberculosa tratada con implante cubierto con nanopartículas de plata. Presentación de un caso
}

\author{
Diego F. Jacob, Joint H. Guimbard Perez, Luis D. E. Orosco Falcone, Juan Carlos Carabajal, Pablo N. Ortiz \\ Departamento de Cirugía Espinal, Servicio de Ortopedia y Traumatología, Sanatorio Allende, Córdoba, Argentina
}

\begin{abstract}
RESUMEN
Se conoce como enfermedad de Gibbus al colapso de la porción anterior de uno o más cuerpos vertebrales que provoca una cifosis segmentaria de ángulo agudo. En general, este tipo de deformidades son producto de infecciones tuberculosas. Uno de los principales problemas que trae apareado esta deformidad es la compresión medular. En el caso presentado, el paciente sufrió esta enfermedad como consecuencia de una infección no tuberculosa, con cifosis angular pososteomielitis, tratado con doble vía de abordaje, utilizando implantes recubiertos con nanopartículas de plata. Los resultados clínico-radiológicos fueron muy satisfactorios. Este caso presenta la conjugación de dos temas poco frecuentes en la medicina actual; por un lado, un tipo de deformidad de la columna que, rara vez, se debe a una infección no tuberculosa y, por otro lado, el implante utilizado, recubierto con nanopartículas de plata que, pese a las controversias, ofrece una nueva posibilidad de tratamiento para pacientes con un riesgo aumentado de infección asociada a implantes, y resulta de interés que sea reconocido por los cirujanos ortopedistas, puesto que existe evidencia suficiente para afirmar su capacidad para reducir la formación de biopelículas.
\end{abstract}

Palabras clave: Gibbus; nanopartículas de plata; cifosis segmentaria.

Nivel de Evidencia: IV

Non-tuberculous Gibbus Deformity, Treated With an Implant Coated With Silver Nanoparticles.
Presentation of a Case

\section{ABSTRACT}

Gibbus disease is the collapse of the anterior portion of one or more vertebral bodies that results in acute angle segmental kyphosis. Generally, these types of deformities are caused by tuberculosis infections. One of the main problems associated with this deformity is spinal compression. In this case, the patient presented this condition as a consequence of a non-tuberculous infection, with angular kyphosis after osteomyelitis, treated with a double approach, using implants coated with silver nanoparticles. We obtained very satisfactory clinical and radiological outcomes. This case presents the intersection of two rare topics in current medicine; on the one hand, a type of spinal deformity that rarely occurs as a consequence of a non-tuberculous infection. On the other hand, the implant used, coated with silver nanoparticles. Although there are still controversies in the literature, this implant offers a new possibility of treatment for patients who are at increased risk of implant-related infection, and it is of interest for orthopedic surgeons, since there is sufficient evidence to support its ability to reduce the formation of biofilms.

Key words: Gibbus; silver nanoparticles; segmental kyphosis.

Level of Evidence: IV

\section{INTRODUCCIÓN}

Se conoce como enfermedad de Gibbus al colapso de la porción anterior de uno o más cuerpos vertebrales que provocan una cifosis segmentaria de ángulo agudo. ${ }^{1}$ El término proviene del latín tardío "gibbosus" que significa jorobado. $^{2}$

Recibido el 15-3-2020. Aceptado luego de la evaluación el 29-7-2020 • Dr. DIEGO F. JACOB • dfjacob @ hotmail.es ID https://orcid.org/0000-0001-7484-0617

Cómo citar este artículo: Jacob DF, Guimbard Perez JH, Orosco Falcone LDE, Carabajal JC, Ortiz PN. Deformidad de Gibbus no tuberculosa tratada con implante cubierto con nanopartículas de plata. Presentación de un caso. Rev Asoc Argent Ortop Traumatol 2021;86(6):802-811. https://doi.org/10.15417/issn. 1852-7434.2021.86.6.1074 
En general, este tipo de deformidades son producto de infecciones tuberculosas en la columna vertebral o son secundarias a anomalías congénitas. ${ }^{1}$ También pueden deberse a algunas fracturas, enfermedades metabólicas, como la mucopolisacaridosis, o infecciones congénitas. ${ }^{3}$ Uno de los principales problemas de este cuadro es la progresión de la cifosis, el balance sagital empeora y, en consecuencia, el riesgo de compresión medular es mayor. ${ }^{1}$

A pesar de que existe gran cantidad de información sobre esta deformidad, resulta muy poco común como resultado de una infección no tuberculosa; en nuestra búsqueda bibliográfica, encontramos un solo caso publicado con dicha característica. ${ }^{4}$ El paciente que presentamos sufrió esta enfermedad a causa de una infección asociada a implantes.

Las tasas de infecciones profundas asociadas a la colocación de implantes en la columna vertebral oscilan entre el 2,2\% y el $25 \%,{ }^{5}$ y los microorganismos aislados más comunes son bacterias de baja virulencia, como Staphylococcus epidermidis, que crecen en los implantes formando biopelículas de multicapas conocidas como biofilms o microfilms,${ }^{6}$ lo que les permite a las bacterias defenderse de los tratamientos antibióticos, como así también de la inmunidad del huésped.

Se ha demostrado que la plata posee un efecto antibacteriano efectivo y puede aplicarse en los implantes en forma de nanopartículas para obtener propiedades físicas, químicas y biológicas mejoradas, con una tendencia a la inhibición de la infección en los implantes ortopédicos. ${ }^{8}$ Por estos motivos, las investigaciones continúan y adquieren especial relevancia cuando nos enfrentamos a pacientes con características especiales, como un metabolismo óseo alterado, sistemas inmunes afectados o infecciones activas o en remisión.

Presentamos un caso inusual de cifosis angular pososteomielitis no tuberculosa, tratado con doble vía de abordaje, utilizando implantes recubiertos con nanopartículas de plata.

\section{CASO CLÍNICO}

Hombre de 69 años, sin antecedentes médicos de relevancia, no tabaquista, que asistió a la consulta de un especialista en patología espinal de nuestra institución, por lumbalgia intensa y dolor en la cara anterior de ambos muslos, y debilidad de los miembros inferiores que le impedían la bipedestación y la deambulación confortables, asociados a parestesias.

Como antecedentes, refirió una cirugía instrumentada de columna dorsolumbar practicada dos años antes, en otra institución, donde había sido sometido a una artrodesis de T11-S1 con barras y tornillos transpediculares, que requirió limpieza y desbridamiento quirúrgico a los cinco días por mala evolución de la herida. En esa oportunidad, no se obtuvo aislamiento ni tipificación de germen. A los 19 meses de la instrumentación, se extrajo el implante.

En nuestra institución, se realizó una punción biopsia de T12 bajo tomografía computarizada, de la cual no se obtuvo aislamiento de germen; tras los análisis pertinentes para descartar tuberculosis, se le diagnosticó osteomielitis vertebral no tuberculosa de T12, según el cuadro clínico y los estudios por imágenes. Como el resultado de los cultivos fue negativo, el Servicio de Infectología indicó un tratamiento empírico para cubrir gérmenes grampositivos y bacilos gramnegativos, con una duración adecuada al diagnóstico de osteomielitis, por lo que se le administró ciprofloxacina más minociclina por vía oral, durante seis semanas.

Se lo citó para controles periódicos hasta que, según los parámetros clínicos y bioquímicos, la infección había curado. Al tercer mes de la extracción del implante, con la herida cicatrizada, sin secreciones ni signos de flogosis y ante la persistencia del dolor, se comenzó a planificar una cirugía de corrección de la deformidad.

El paciente estaba en sillón de ruedas, refería lumbalgia asociada con dolor intenso en la cara anterior de ambos miembros inferiores, y el puntaje en la escala analógica visual era de 9/10. Además, tenía debilidad en ambos miembros inferiores, con atrofia muscular marcada en ambos muslos y masas gemelares, podía caminar con ayuda, pero sentía dolor, los signos mielopáticos estaban ausentes, los reflejos osteotendinosos profundos estaban conservados y la fuerza muscular de miembros inferiores era de 3/5 según la escala del Medical Research Council. A la inspección, se observó un desequilibrio sagital positivo severo, el puntaje del Oswestry Disability Index era del $75 \%$, que corresponde a la categoría de "discapacidad".

Se solicitaron estudios por imágenes. El espinograma de pie mostró un desequilibrio sagital global. Con el sistema SurgiMap®, se midieron los parámetros espinopélvicos y se obtuvieron los siguientes resultados: eje vertical sagital (sagital vertical axis, SVA) muy positivo de $229 \mathrm{~mm}$, con un ángulo T1-pelvis (T1-pelvis angle, TPA) de $57^{\circ}$, incidencia pélvica (pelvic incidence, PI) $86^{\circ}$, inclinación pélvica (pelvic tilt, $\mathrm{PT}$ ) $45^{\circ}$, pendiente sacra (sacral slope, SS) $41^{\circ}$, cifosis torácica (thoracic kyphosis, TK) $60^{\circ}$, lordosis lumbar (lumbar lordosis, LL) $53^{\circ}$, PI-LL $33^{\circ}$, GT $66^{\circ}$ y gap de 12 . El paciente tenía una deformidad de Gibbus a expensas del acuñamiento anterior de T12, con cifosis segmentaria de T11-L1 de $111^{\circ}$ (Figura 1). 

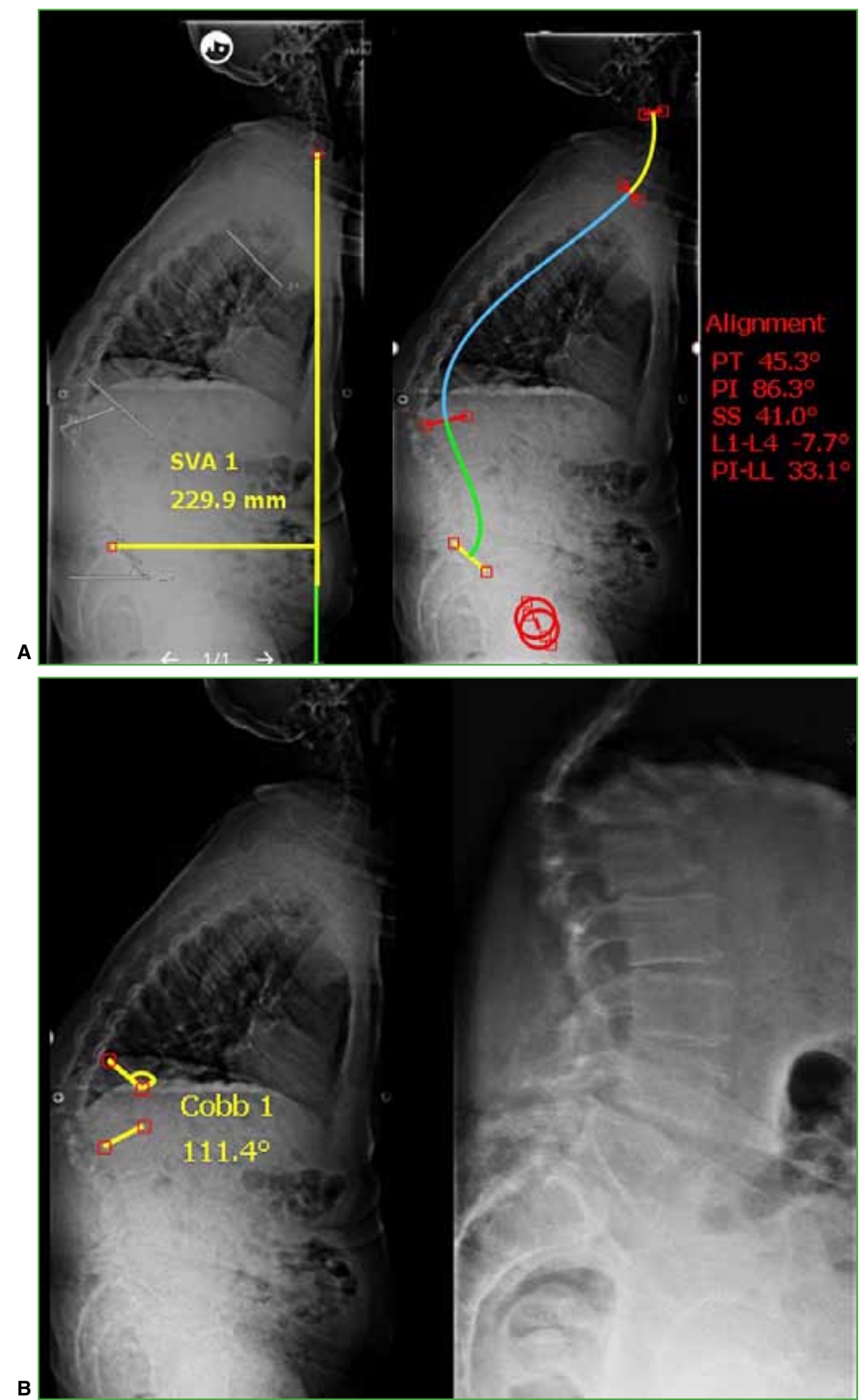

Figura 1. Espinograma de perfil de pie antes de la cirugía. A. Se observa el valor del eje vertical sagital. B. Parámetros espinopelvianos y curvas vertebrales. 
En la tomografía computarizada, se observó una osteólisis $>50 \%$ del cuerpo de T12 a predominio anterior y pérdida de stock óseo en el platillo vertebral superior de L1 más signos secuelares de aflojamiento de tornillos en todos los niveles restantes, principalmente en el hueso sacro (Figura 2).

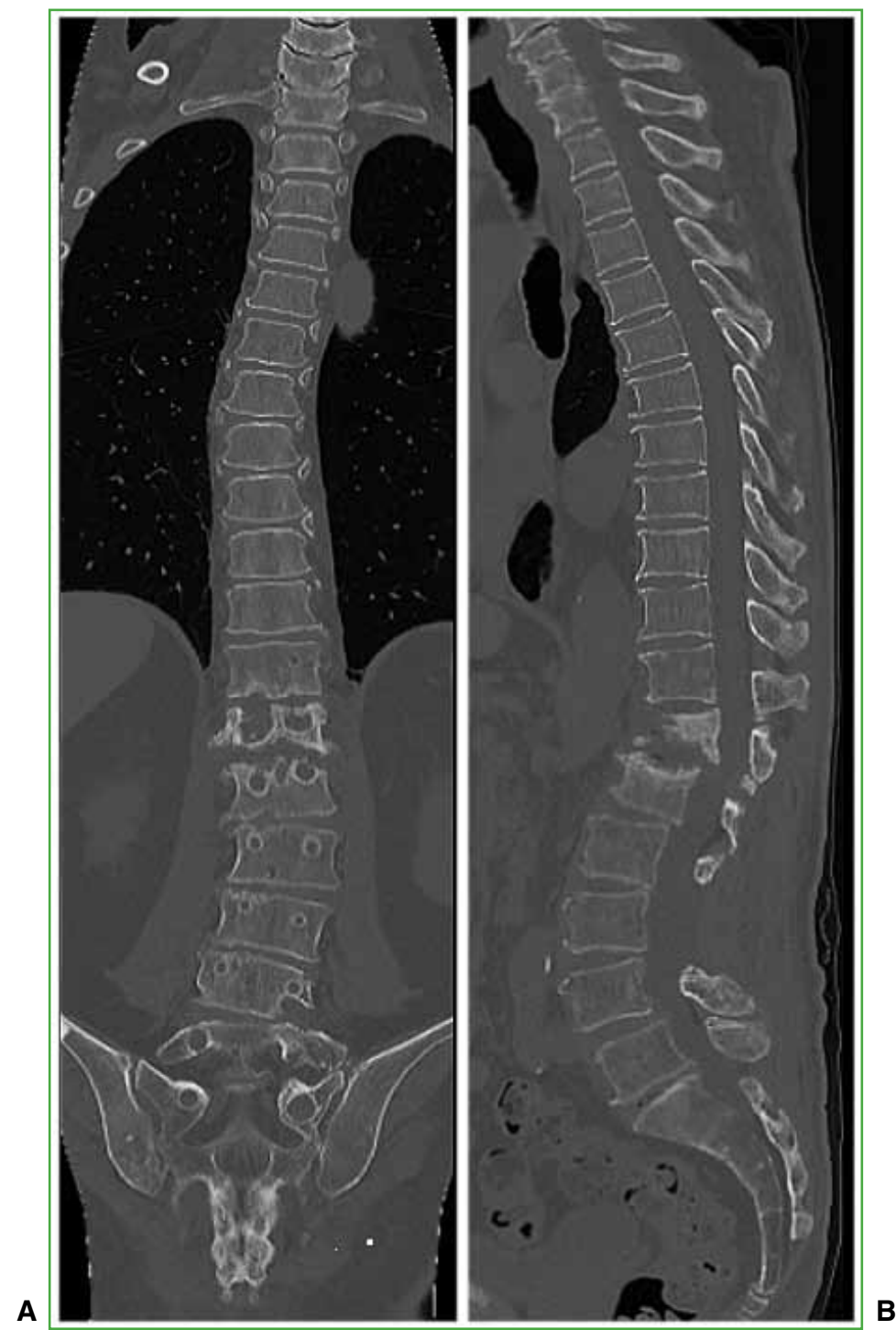

Figura 2. Tomografía computarizada de columna dorsolumbosacra.

A. Osteólisis a predominio de las vértebras superiores de la instrumentación y el sacro. B. Destrucción de la vértebra T12 con gran acuñamiento anterior.

En la resonancia magnética, se visualizó el estrechamiento del canal espinal en T12-L1, con hiperintensidad en la secuencia T2 del disco a dicho nivel, asociado con la destrucción de ambos cuerpos vertebrales (Figura 3). Se realizó, además, un centellograma óseo que mostró hipercaptación leve en T12-L1. Los parámetros bioquímicos indicaban que no había infección activa. 


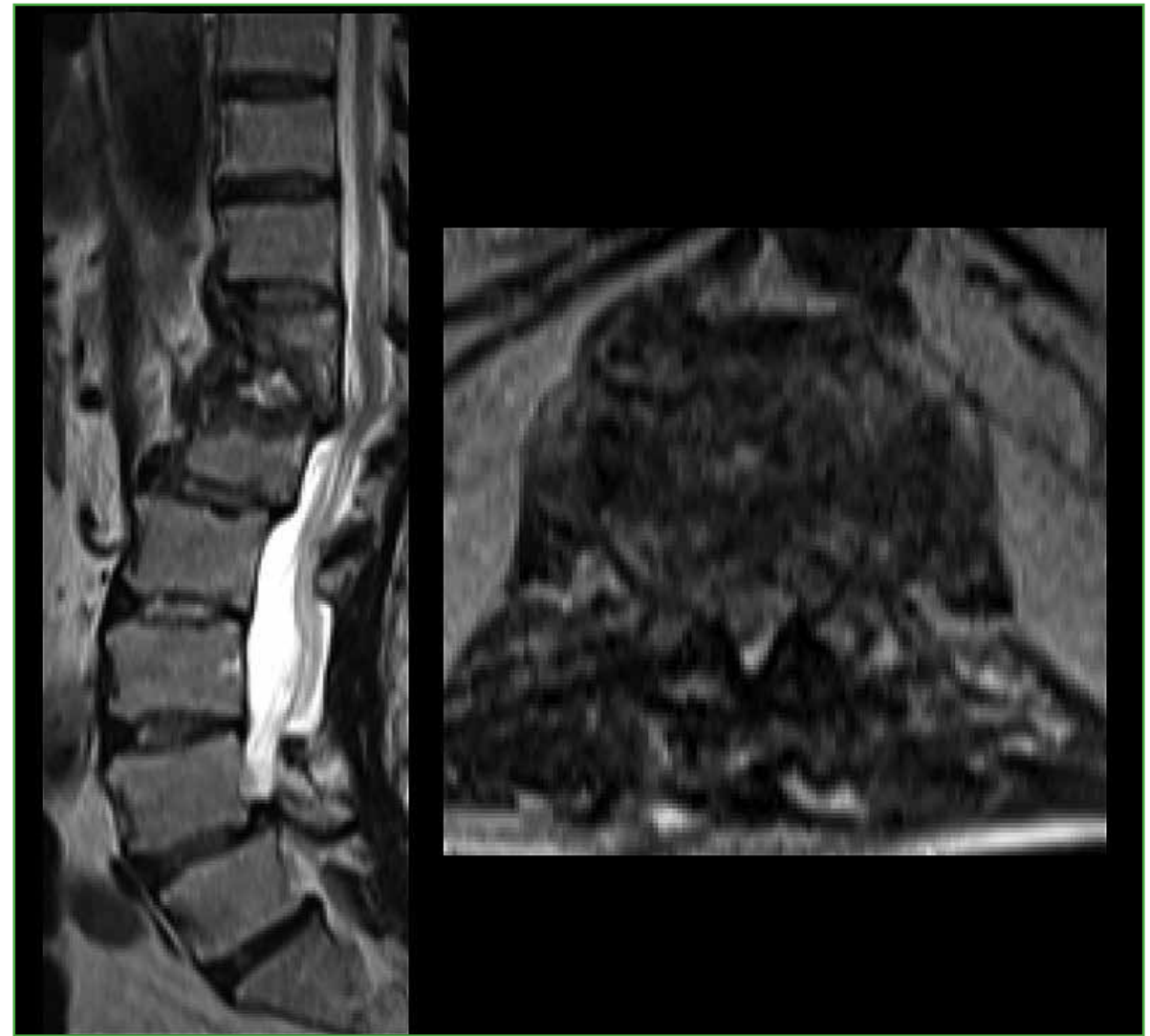

Figura 3. Resonancia magnética de columna dorsolumbosacra que muestra la estrechez resultante del canal medular.

\section{Técnica quirúrgica}

Se realizó un primer tiempo quirúrgico anterior por toracotomía, con exéresis de la 12va costilla y corporectomía de T12, y colocación de caja telescopada autoexpandible con injerto óseo autólogo de costilla y tornillos laterales al cuerpo de T11 y L1 unidos con una barra de titanio, todos recubiertos con nanopartículas de plata para prevenir la formación de biopelículas. Siete días después, se llevó a cabo el segundo tiempo quirúrgico posterior, bajo monitoreo neurofisiológico, con artrodesis de T4 a pelvis mediante tornillos pediculares y tres barras de titanio con corrección de la deformidad y la colocación de injerto óseo autólogo más injerto heterólogo de banco, más descompresión y liberación mielomenigorradicular de L4-L5.

El paciente toleró bien el procedimiento y fue dado de alta deambulando sin dolor con andador de cuatro puntos, a los cinco días.

Se realizaron controles evolutivos periódicos durante el primer mes, a los tres y seis meses; en la actualidad, a los ocho meses de la intervención, la herida está cicatrizada y el paciente camina sin ayuda, sin dolor en la región dorsolumbar y dolor leve en los miembros inferiores (puntaje 2/10 en la escala analógica visual). Ha completado el plan de neurorrehabilitación para fortalecimiento muscular y perfeccionamiento de la marcha, con un puntaje del Oswestry Disability Index del 20\%, que corresponde a la categoría de "limitación funcional moderada" (Figura 4). Tanto el paciente como sus familiares están muy satisfechos con los resultados obtenidos. El paciente otorgó el consentimiento informado por escrito para la publicación del caso. 


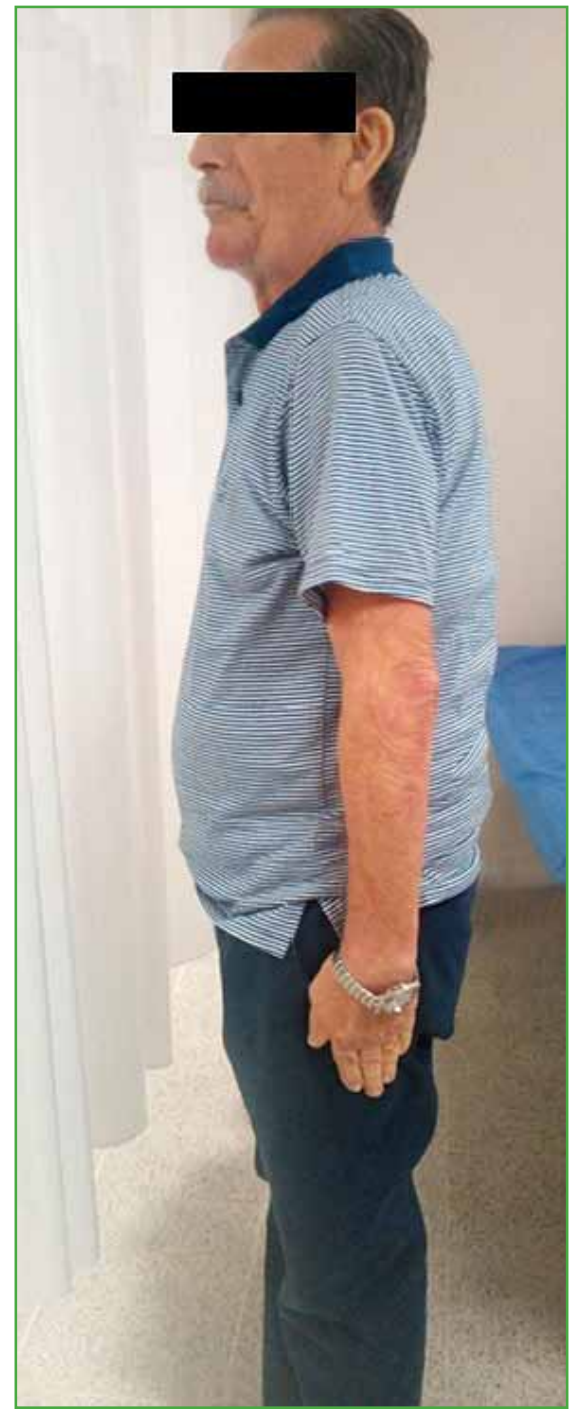

Figura 4. Imagen posoperatoria. Paciente de perfil.

En las nuevas mediciones con el programa SurgiMap®, en el espinograma de pie, se observa un SVA positivo de $35 \mathrm{~mm}$ y una cifosis segmentaria T11-L1 de $11^{\circ}$, con TPA $27^{\circ}$, PI $74^{\circ}$, PT $34^{\circ}$, SS $40^{\circ}$, TK $37^{\circ}$, LL $49^{\circ}$, PI-LL $24^{\circ}$, GT $29^{\circ}$ y gap de 8, con mejoría en todos los parámetros espinopélvicos (Figura 5).

Por otro lado, en la tomografía computarizada, se puede visualizar la consolidación ósea en todos los planos y la invasión de hueso neoformado alrededor del implante intersomático (Figura 6). 


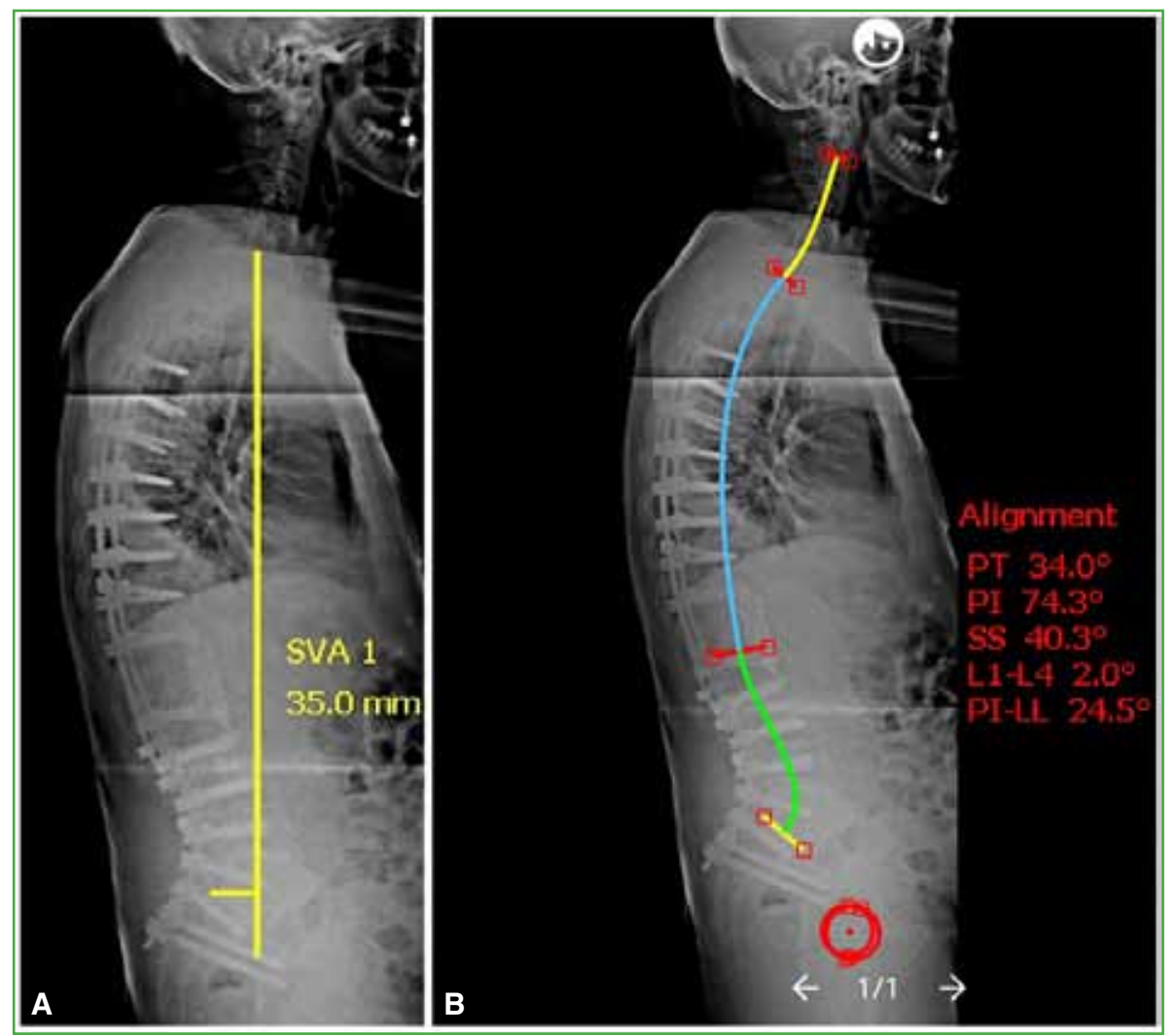

Figura 5. Espinograma de perfil de pie después de la cirugía. A. Valor del eje vertical sagital resultante. B. Parámetros espinopélvicos y curvas vertebrales obtenidos.

Figura 6. Tomografía computarizada de columna dorsolumbosacra posoperatoria. Se observa el proceso de consolidación ósea.

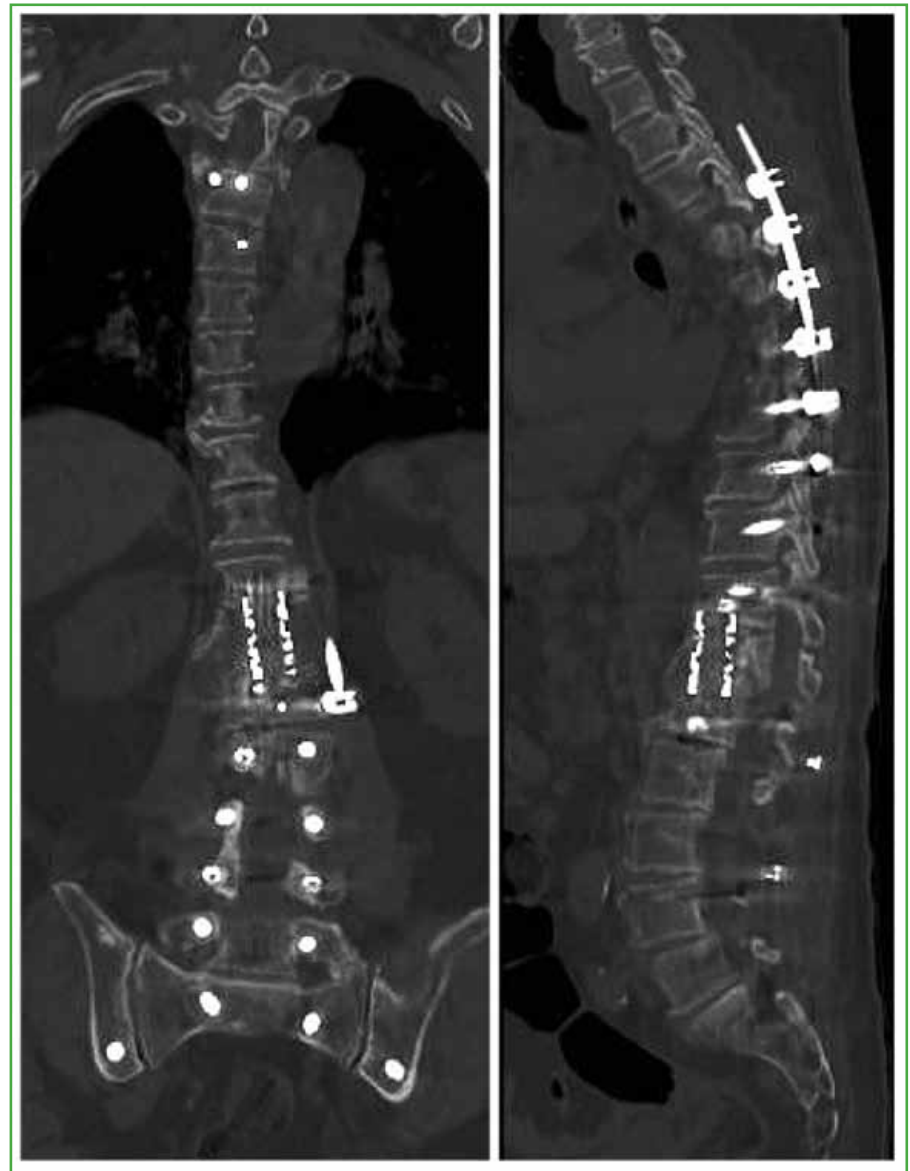




\section{DISCUSIÓN}

Los objetivos del tratamiento quirúrgico de la cifosis angular incluyen la mejoría de la clínica neurológica y de los déficits por compresión neural, la restauración de la alineación normal mediante la corrección de la deformidad y estabilización de la columna vertebral con una artrodesis. ${ }^{9}$ Como se puede observar en las mediciones radiológicas descritas, la incidencia pélvica posoperatoria descendió $12^{\circ}$ respecto de la inicial, lo que puede resultar llamativo teniendo en cuenta que dicho parámetro ha sido descrito tradicionalmente como "estático". Sin embargo, estudios recientes han demostrado que la incidencia pélvica puede variar con la edad, el índice de masa corporal o tras algunos procedimientos espinales, ${ }^{10,11} \mathrm{e}$ inclusive ante las posiciones de flexión y extensión, esto último parece deberse a la movilidad del sacro con respecto a la pelvis a través de la articulación sacroilíaca, evidenciado por cambios desproporcionados en la pendiente sacra versus la inclinación pélvica. ${ }^{12}$

Para conseguir el éxito terapéutico, es más importante obtener una artrodesis sólida que lograr una corrección anatómica de la deformidad. Y, basándose en esta máxima, la importancia del soporte anterior y del injerto óseo es clara, y ha sido bien documentada. ${ }^{13} \mathrm{~A}$ pesar del avance de las técnicas quirúrgicas para la estabilización anterior y la descompresión posterior, el procedimiento quirúrgico ideal continúa siendo controvertido.

En aquellas inestabilidades espinales donde las columnas anterior, media y posterior están afectadas, independientemente de la patología que las origina, la descompresión exitosa, la corrección de la deformidad y la estabilización requieren procedimientos anteriores y posteriores. ${ }^{14}$

El advenimiento de dichas técnicas quirúrgicas de complejidad ascendente genera, a su vez, la necesidad de contar con implantes de características mecánicas y biológicas cada vez más exigentes, con capacidad de durar mucho tiempo, resistencia mecánica, escasos o nulos efectos adversos sobre el huésped, relativa resistencia a la infección, costos accesibles para el sistema de salud, disponibilidad y acceso de la población.

Estos requerimientos han dado lugar a nuevos paradigmas, en los que las nuevas características resultantes de los biomateriales no dependen necesariamente del descubrimiento de nuevos materiales con propiedades únicas, sino que son más bien la resultante del uso inteligente y combinado de materiales ya disponibles.

En este contexto, podemos afirmar que la actividad antimicrobiana de la plata se conoce muy bien; por lo tanto, el estudio de este metal en la industria de los implantes ortopédicos ha tomado auge en el último tiempo. Su actividad bactericida se basa en la inactivación de enzimas clave, como la succinato deshidrogenasa por unión de grupos tiol, la formación de radicales hidroxilo y el posterior daño del ADN.

En un estudio de van Hengel y cols. ${ }^{15}$ cuyo objetivo fue desarrollar implantes metálicos equipados con funcionalidad antimicrobiana para evitar la infección asociada, la superficie de los implantes se "biofuncionalizó" utilizando oxidación electrolítica plasmática en presencia de nanopartículas de plata. Se eligieron las nanopartículas de plata, porque, cuando se oxidan, liberan iones plata que, se sabe, son agentes antimicrobianos potentes y han demostrado un fuerte comportamiento bactericida contra un amplio espectro de bacterias que incluyen Staphylococcus aureus resistente a meticilina a través de múltiples mecanismos, como el daño a las membranas bacterianas y la producción de especies reactivas de oxígeno. ${ }^{16}$

En este estudio, los implantes se incubaron con $S$. aureus resistente a meticilina durante $48 \mathrm{~h}$, en condiciones que inducen la formación de biopelículas. Después de $48 \mathrm{~h}$ de incubación, en los implantes del grupo de control, se formaron biopelículas, las bacterias se apilaban cada vez más una encima de la otra para formar hasta 4 o 5 capas. En cambio, en los implantes con plata, menos bacterias se unieron a la superficie de los implantes y no se observaron signos de formación de biopelículas.

Tras una extensa búsqueda, no hemos encontrado en la bibliografía actual series de casos, como así tampoco reportes de casos donde se hayan utilizado implantes de estas características en la cirugía espinal. Sin embargo, en 2016, Hazer y cols. publicaron un estudio en conejos que ha logrado resultados prometedores respecto de la inhibición de la biopelícula en implantes espinales recubiertos con nanopartículas de plata; ${ }^{17}$ a su vez, Hegde y cols., en un estudio reciente en ratones, llegaron a conclusiones similares. ${ }^{18}$

Hay investigaciones que demuestran que las nanopartículas de plata y la plata iónica poseen un efecto antibacteriano a concentraciones que no son citotóxicas para las células eucariotas. ${ }^{19}$ Sin embargo, algunos autores afirman que los implantes con plata pueden estar asociados con toxicidad, causada por los altos niveles de iones plata. ${ }^{20} \mathrm{En}$ general, podemos decir que las células de los mamíferos, que poseen un mayor tamaño, una organización estructural y funcional superior, y más capacidad de producir matriz extracelular, son más tolerables a la plata que las células bacterianas. ${ }^{21}$ 
Los resultados a corto plazo de las aplicaciones de estos implantes de plata no mostraron ningún efecto adverso sobre las funciones hepáticas y renales del huésped. ${ }^{22}$ Sin embargo, a nivel celular, los impactos de los iones plata en la viabilidad y diferenciación de las células óseas aún no se han investigado en detalle.

Es por esto que, en la actualidad, crecen las preocupaciones con respecto a los efectos secundarios citotóxicos de la plata iónica y las nanopartículas de plata en células de mamíferos y sistemas de órganos. ${ }^{23}$

En un estudio de Albers y cols., se demostró que las partículas de plata provocan efectos citotóxicos en osteoblastos y osteoclastos. ${ }^{20}$

Por otra parte, estudios previos ya habían demostrado que tanto la plata iónica como las nanopartículas de plata causan efectos citotóxicos en diferentes sistemas in vitro e in vivo. ${ }^{24}$ No hay consenso sobre la toxicidad de estas partículas para los seres humanos, la mayoría de las investigaciones sobre la toxicidad de las nanopartículas de plata se basan en experimentos celulares in vitro y experimentos con animales a relativamente corto plazo.

\section{CONCLUSIONES}

Este caso presenta la conjugación de dos temas poco frecuentes en la medicina actual; por un lado, la presentación de un tipo de deformidad de columna que, rara vez, ocurre como consecuencia de una infección no tuberculosa, cuyo tratamiento aún sigue siendo controvertido desde el punto de vista biomecánico. Comunicamos aquí un método terapéutico que, hasta el momento, logra un excelente resultado clínico para el paciente, con la restauración de la bipedestación, la marcha armónica, y gran mejoría del dolor y los síntomas neurológicos. Por otro lado, el implante utilizado, de última generación, recubierto con nanopartículas de plata. Si bien aún se encuentra en estudio y, para algunos autores, su uso resulta controvertido por los motivos antes descritos, ofrece una nueva posibilidad de tratamiento para pacientes con un riesgo más alto de infección asociada con los implantes. Resulta de interés que sea reconocido y tenido en cuenta por los cirujanos ortopedistas, porque hay evidencia que avala su capacidad para reducir la formación de biopelículas.

Conflicto de intereses: Los autores no declaran conflictos de intereses.

\section{BIBLIOGRAFÍA}

1. Ghandi M, Aycock RD, Berwald N, Hahn B. Gibbus deformity. J Emerg Med 2015;49(3):340-1. https://doi.org/10.1016/j.jemermed.2015.04.004

2. Merriam-Webster. Gibbous. En: Merriam-Webster.com dictionary. Disponible en: https:// www.merriam-webster.com/dictionary/gibbous

3. Palmucci S, Attinà G, Lanza ML, Belfiore G, Cappello G, Foti PV, et al. Imaging findings of mucopolysaccharidoses: a pictorial review. Insights Imaging 2013;4(4):443-59. https://doi.org/10.1007/s13244-013-0246-8

4. Seksenyan A, Esfahani DR, Hu K, Mehta AI. Gibbus deformity after non-tuberculosis osteomyelitis. J Spine Surg 2017;3(4):736-9. https://doi.org/10.21037/jss.2017.12.02

5. Maciejczak A, Wolan-Nieroda A, Wałaszek M, Kołpa M, Wolak Z. Antibiotic prophylaxis in spine surgery: a comparison of single-dose and 72-hour protocols. J Hosp Infect 2019;103(3):303-10. https://doi.org/10.1016/j.jhin.2019.04.017

6. Götz F. Staphylococcus and biofilms. Mol Microbiol 2002;43(6):1367-78. https://doi.org/10.1046/j.1365-2958.2002.028276.x

7. Kristian SA, Birkenstock TA, Sauder U, Mack D, Götz F, Landmann R. Biofilm formation induces C3a release and protects Staphylococcus epidermidis from IgG and complement deposition and from neutrophil-dependent killing. $J$ Infect Dis 2008;197(7):1028-35. https://doi.org/10.1086/528992 
8. Qing Y, Cheng L, Li R, Liu G, Zhang Y, Tang X, et al. Potential antibacterial mechanism of silver nanoparticles and the optimization of orthopedic implants by advanced modification technologies. Int J Nanomedicine 2018;13:331127. https://doi.org/10.2147/IJN.S165125

9. Jung-Hee Lee, Hyun-Seok Oh, Jeong-Gyu Choi. Comparison of the posterior vertebral column resection with the expandable cage versus the nonexpandable cage in thoracolumbar angular kyphosis. Clin Spine Surg 2017;30(4):E398-E406. https://doi.org/10.1097/BSD.0000000000000236

10. Iyer S, Lenke LG, Nemani VM, Albert VM, Sides A, Metz LN, et al. Variations in sagittal alignment parameters based on age: a prospective study of asymptomatic volunteers using full-body radiographs. Spine (Phila $\mathrm{Pa}$ 1976) 2016;41(23):1826-36. https://doi.org/10.1097/BRS.0000000000001642

11. Noshchenko A, Hoffecker L, Cain CMJ, Patel VV, Burger EL. Spinopelvic parameters in asymptomatic subjects without spine disease and deformity: a systematic review with meta-analysis. Clin Spine Surg 2017;30(9):392-403. https://doi.org/10.1097/BSD.0000000000000533

12. Schroeder N, Noschenko A, Burger E, Patel V, Cain C, Ou-Yang D, et al. Pelvic incidence changes between flexion and extension. Spine Deform 2018;6(6):753-61. https://doi.org/10.1016/j.jspd.2018.03.008

13. Winter RB. Congenital scoliosis. Clin Orthop Relat Res 1973;93:75-94. Disponible en: https://journals.lww.com/clinorthop/Citation/1973/06000/Congenital_Scoliosis.10.aspx

14. Böhm H, Harms J, Donk R, Zielke K. Correction and stabilization of angular kyphosis. Clin Orthop Relat Res 1990;(258):56-61. PMID: 2394059

15. van Hengel IAJ, Riool M, Fratila-Apachitei LE, Witte-Bouma J, Farrell E, Zadpoor AA, et al. Selective laser melting porous metallic implants with immobilized silver nanoparticles kill and prevent biofilm formation by methicillin-resistant Staphylococcus aureus. Biomaterials 2017;140:1-15. https://doi.org/10.1016/j.biomaterials.2017.02.030

16. Rizzello L, Pompa PP. Nanosilver-based antibacterial drugs and devices: mechanisms, methodological drawbacks, and guidelines. Chem Soc Rev 2014;43(5):1501-18. https://doi.org/10.1039/c3cs60218d

17. Hazer DB, Sakar M, Dere Y, Altınkanat G, Ziyal MI, Hazer B. Antimicrobial effect of polymer-based silver nanoparticle coated pedicle screws: experimental research on biofilm inhibition in rabbits. Spine (Phila Pa 1976) 2016;41(6):E323-9. https://doi.org/10.1097/BRS.0000000000001223

18. Hegde V, Park HY, Dworsky E, Zoller SD, Xi W, Johansen DO, et al. The use of a novel antimicrobial implant coating in vivo to prevent spinal implant infection. Spine (Phila Pa 1976) 2020;45(6):E305-E311. https://doi.org/10.1097/BRS.0000000000003279

19. Amin Yavari S, Loozen L, Paganelli FL, Bakhshandeh S, Lietaert K, Groot JA, et al. Antibacterial behavior of additively manufactured porous titanium with nanotubular surfaces releasing silver ions. ACS Appl Mater Interfaces 2016;8(27):17080-9. https://doi.org/10.1021/acsami.6b03152

20. Albers CE, Hofstetter W, Siebenrock KA, Landmann R, Klenke FM. In vitro cytotoxicity of silver nanoparticles on osteoblasts and osteoclasts at antibacterial concentrations. Nanotoxicology 2013;7(1):30-6. https://doi.org/10.3109/17435390.2011.626538

21. Chernousova S, Epple M. Silver as antibacterial agent: ion, nanoparticle, and metal. Angew Chem Int Ed Engl 2013;52(6):1636-53. https://doi.org/10.1002/anie.201205923

22. Hardes J, Ahrens H, Gebert C, Streitbuerger A, Buerger H, Erren M, et al. Lack of toxicological side-effects in silver-coated megaprostheses in humans. Biomaterials 2007;28(18):2869-75. https://doi.org/10.1016/j.biomaterials.2007.02.033

23. Ahamed M, Alsalhi MS, Siddiqui MKJ. Silver nanoparticle applications and human health. Clin Chim Acta 2010;411(23-24):1841-48. https://doi.org/10.1016/j.cca.2010.08.016

24. Asharani PV, Lianwu Y, Gong Z, Valiyaveettil S. Comparison of the toxicity of silver, gold and platinum nanoparticles in developing zebrafish embryos. Nanotoxicology 2011;5(1):43-54.

https://doi.org/10.3109/17435390.2010.489207 